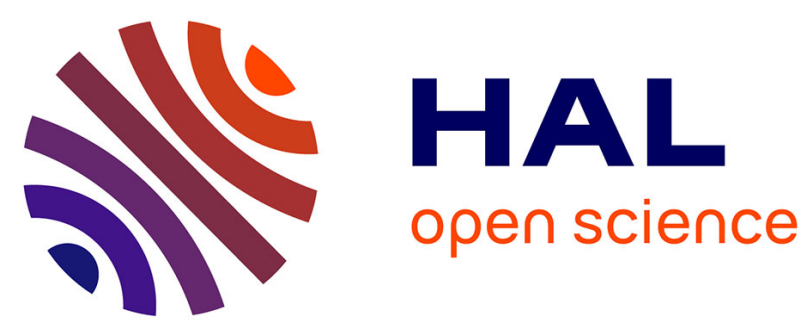

\title{
[Appendice II] Géologie, chronologie et palynologie de la station tardenoisienne de Coincy (Aisne)
}

Henriette Alimen, Georgette Delibrias, Jacqueline Sauvage

\section{To cite this version:}

Henriette Alimen, Georgette Delibrias, Jacqueline Sauvage. [Appendice II] Géologie, chronologie et palynologie de la station tardenoisienne de Coincy (Aisne). Gallia Préhistoire - Fouilles et monuments archéologiques en France métropolitaine, 1964, Gisements tardenoisiens de l'Aisne, 7, pp.95-106. 10.3406/galip.1964.2187 . hal-02402191

\section{HAL Id: hal-02402191 \\ https://hal.science/hal-02402191}

Submitted on 26 Oct 2021

HAL is a multi-disciplinary open access archive for the deposit and dissemination of scientific research documents, whether they are published or not. The documents may come from teaching and research institutions in France or abroad, or from public or private research centers.
L'archive ouverte pluridisciplinaire HAL, est destinée au dépôt et à la diffusion de documents scientifiques de niveau recherche, publiés ou non, émanant des établissements d'enseignement et de recherche français ou étrangers, des laboratoires publics ou privés.

\section{(ㅇ)(1) $\$$}

Distributed under a Creative Commons Attribution - NonCommercial - NoDerivatives $\mid 4.0$ 
Appendice II. Géologie, chronologie et palynologie de la station tardenoisienne de Coincy (Aisne)

Henriette Alimen, Georgette Délibrias, J. Sauvage

Citer ce document / Cite this document :

Alimen Henriette, Délibrias Georgette, Sauvage J. Appendice II. Géologie, chronologie et palynologie de la station tardenoisienne de Coincy (Aisne). In: Gallia préhistoire, tome 7, 1964. pp. 95-106;

doi : https://doi.org/10.3406/galip.1964.2187

https://www.persee.fr/doc/galip_0016-4127_1964_num_7_1_2187

Fichier pdf généré le 12/11/2020 
APPENICH: II

\section{GEOLOGIE, CHRONOLOGIE ET PALYNOLOGIE DE LA STATION TARDENOISIENNE DE COINCY (Aisne)}

La station de Coincy, dans le Tardenois, ou fut pris le type de l'industrie tardenoisienne. ayant donné lieu récemment a des foulles conduites par J. IIsoct (J. Insor'T. 1964), nous avons visité (') gisement le 7 juillet 1961, afin d'y faire observations et prélevements. nolamment aux lieux-dits la Chambre des Fées el le Marécage-sous-le-Ciéanl.

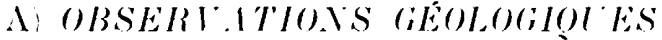

les observalions géologiques essentielles ont ete faites a la Chambre des fées.

\section{Stratigraphie de la slation à la Chambre des Fies.}

Xous appellerons coupe I celle où nous avons vu la stratigraphie la plus complete (tig. I).

Celle coupe était située en N3 sur le plan horizontal donné par .J. Hivort (1964, fig. 2). el orientie perpendiculairement à la pente. D'une hauteur totale de $1,50 \mathrm{~m}$, elle était occupée sur les $0,80 \mathrm{~m}$ supérieurs par un podzol (couches AI. A2, et B). La couche archéologique, ou les silex se mèlaient a des fragments rocheux, y élait située vers le haul de la couche noire. marbrée de jaune. zone de. roncentration de l'humus et des oxydes de fer (couche 3). La moitié inférieure de la coupe présentait

II tes sables orre veinés horizontalement de lits bruns (couche $\mathrm{C}$ ) visibles sur $0,70 \mathrm{~m}$.

line seconde coupe (coupe II). située au voisinage de la précédente, mais orienté selon la pente (fig. 2). montrait une disposilion générale analogue, modifiée par la présence d'un bloc Ie gris (i, enfoui sous un podzol aux horizons mieux marqués que dans la coupe $I\left(A_{0}, \lambda_{1}, A_{2}, B_{1}\right.$, $\mathrm{B}_{2}$ ). Ce bloc fossilisait la couche zonćc (couche $\mathrm{C}$ ). Au sein de celle-ci, éparses aulour de la base du bloc gréseux, des plaquettes de gris s'étalaient en une couche horizontale au niveau l'. I'autres

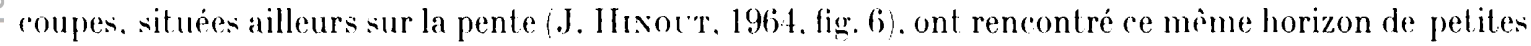
plaques gréseuses, loujours au sein de la rouche zonée et au voisinage de blocs de grris enfouis (photo lig. 7 .

Rappelons enfin que. si la couche a industrie tardenoisienne 'T, se trouve au sommet de l'horizon aliolique dans les coupes I et 11, par contre là où l'alios n'est pas assez développé pour avoir eflacé les zébrures dans la partie haute de la zonation. on rencontre l'industrie au sein des sables zébrés, dans leur partie haute, toujours plus haul que l'horizon a plaques gréseuses P :voir la fig. fi de l'étude de. J. Hinotet).

\section{Conche $P$ à accumulation de fragments rochent.}

Jes fragments rocheux de la couche $P$ sont uniquentent grexeux. Ils comprennent, en assez. grande quantité, des plaques polygonales. mesurant 10 a $16 \mathrm{~cm}$ de long. selon leur plus grande dimension, épaisses de 2 a $;$ cm. Elles sont bombées sur une de leurs faces que nous appellerons supérieure. Cette face porte sur sa totalité ou partiellement, une patine gris foncé, analogue à celle de la surface des gros blocs épars sur le grisement. Cette patine est épaisse de $1 \mathrm{~cm}$. Les faces latérales de ces plapues sont sensiblemenl reclilignes el planes. el ne portent la paline grise qu'au voisinage de la face bombée. La fare inférieure. lorsquelle est inlacte. est revelue d'une patine gris fonce. mais tris peu épaisse (2 mm seulement ; en oul re. celle face est légérement éolisée. bille porte des rupules de cryoclastisme qui, elles. ne sont revetues d'aucune patine. 


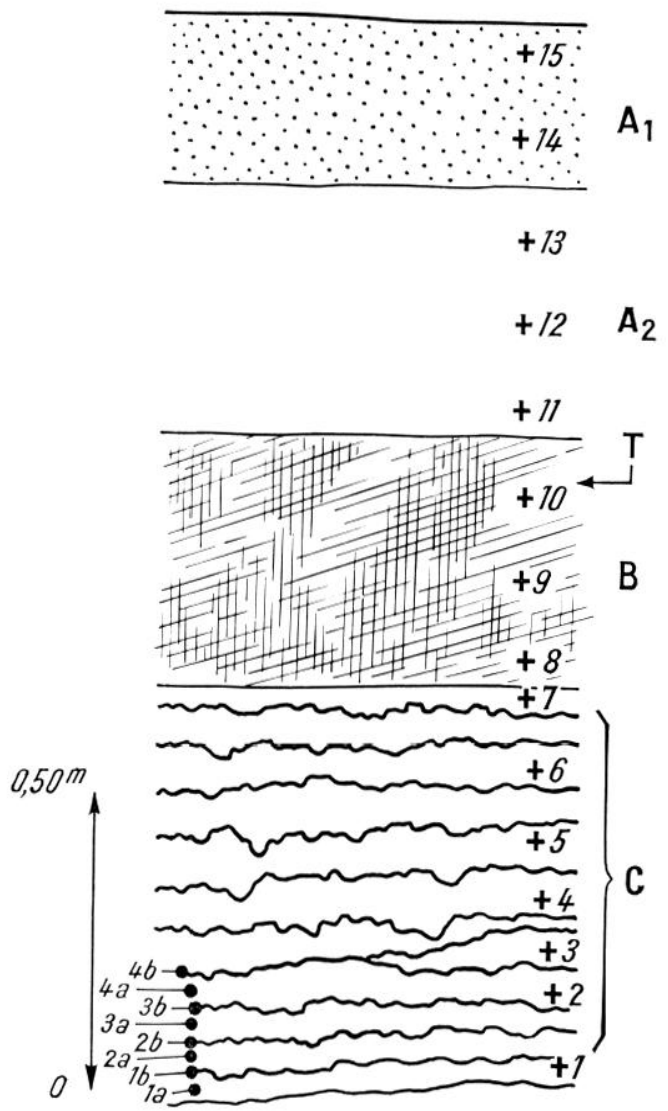

1. Coincy, Chambre des Fies, coupe I. I.es divers horizons du podzol $\left\{A_{1}, A_{2}\right.$ et $\left.B\right\}$ surmontent la couche $C^{-}$de sables gris-jaune, zibris horizonlalement de lits bruns. I.es croix et les numeros, i droite de la figure, marquent les prélevements effectués en vue de l'analyse pollinique. Sur le coli gauche de la coupe sont placés des points noirs figurant la position stratigraphique des prélivements faits en vue de l'analyse pétrographique. lin réalité, ceux-ci ont été pris dans une coupe voisine équivalente. $\mathrm{l}$ : emplacement de l'industrie tardenoisienne.

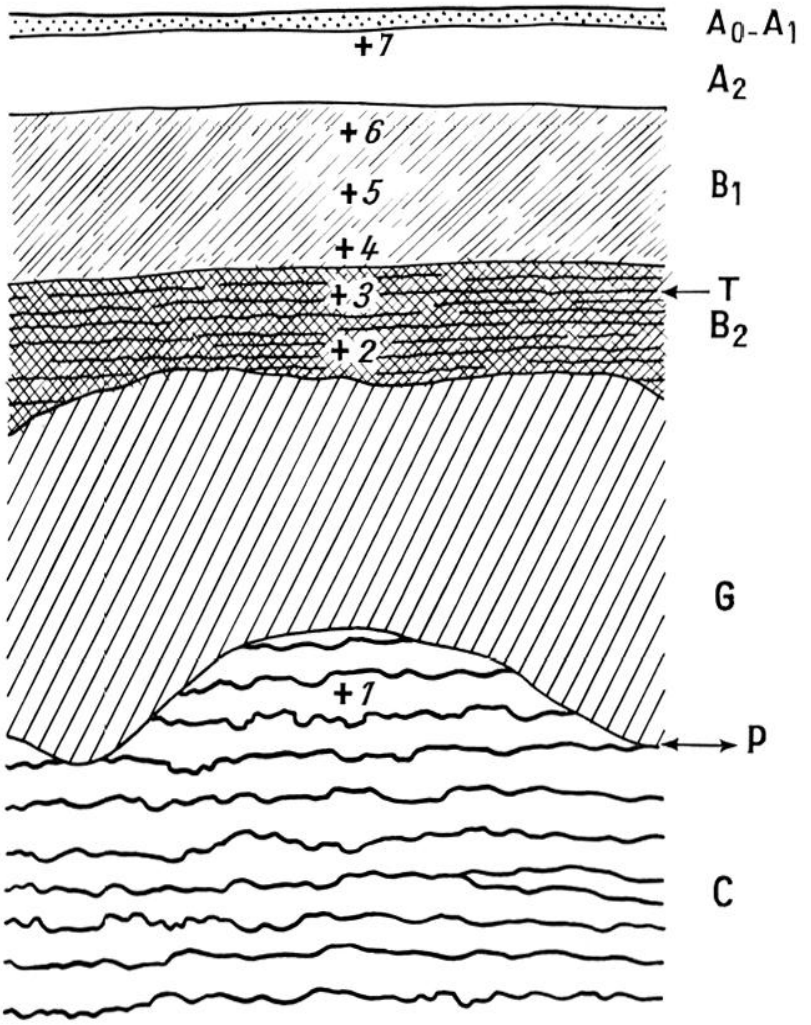

2. Coincy-chambre des Fées, conpe II. $\Lambda_{0}-A_{1}, \Lambda_{2}, B_{1}$ ef $B_{2}$ : divers horizons du podzol. a : gros bloc de gress l: : couche zonce analogue a celle de la tig. 1. P' : posilion des plaquetles de grés. 'T' : siluation de l'industrie lardenoisienne. less crois at les mumíros marquent les prélivements effectuis pour l'analyse pollinique.

Ces plaques polygonales sont manifestement dues a l'exfoliation, sous des causes naturelles, des polygones bombés qui découpent en "peau de crocodile " la surface des blocs de gries auversiens. line telle morphologie des blocs de grès a été anciennement signalée dans le Bassin de Paris et récemment décrite en détail sur les blocs de grès stampiens de la Forìt de Fontainebleau (M. Bнoc:H, 19\%i)). Or, des blocs i peau de crocodile s'observent sur les gress de Coincy, et précisément sur le gisement mème de la Chambre des Fées. Les dimensions et la forme des plaquettes correspondent parfaitement. à celles des polygones de la peau de crocodile. Innfin l'ensemble des fouilles a révélé que les plaqueltes sont localisées sur le pourtour des blocs de grès enfouis dans le sable'.

L'exfoliation de ces polygones ne peut ètre imputable qu'au cryoclastisme. Actuellement on n'observe aucune tendance vers un tel phénomene, sur aucun de très nombreux blocs à peau de

(1) Renseignement oral de M. J. Hrxort. 
crocodile du stampien et de l'Auversien du Bassin parisien. Leur détachement des blocs de griss évoque donc une période de froid intense. Cette période est antérieure à l'enfouissement des plaques dans la couche $C$. puisque la face d'éclatement des plaques a eu le temps de prendre patine et éolisation. Enfin, les traces de cryoclastisme de la face inférieure des plaques, dépourvues de patine et, d'éolisation, doivent itre contemporaines de l'epoque d'enfouissement des plaques.

Entre ces plaques de grande taille existent de petits fragments de diverses formes. Certains d'entre eux. bien que globuleux, ont mème origine que les plaquettes. Ils proviennent de grès à peau de crocodile de petites mailles (亏 à $6 \mathrm{~cm}$ ). La plupart sont en grès lendre et leurs arêtes, de ce fait, sont très émoussées. D'autres, plats el minces, sont des écailles de gel, provenant des plaques. Leur diamètre peut atleindre exceptionnellement 8 a $10 \mathrm{~cm}$. mais leur épaisseur ne dépasse pas $1 \mathrm{~cm}$ vers le centre, où l'ombilic caractéristique des écailles de cryoclastisme reste bien marqué.

Enfin, il y a de petits fragments provenant de la fracturation d'écailles minces sans qu'on y discerne aucune trace de chocs intentionnels.

La succession des faits parait donc ètre la suivanle. Au cours d'un moment tris froid, les polygones de la peau de crocodile des blocs de grès ont été exloliés et se sont accumulés autour des blocs. Ils sont restés exposés à l'air. pendant une période d'interruption de la sédimentation, correspondant peut être à une légère rémission du climat, acquérant sur les faces d'éclatement la légère patine et la faible éolisalion que nous avons signalées, puis ils ont subi de nouveaux phénomènes d'éclatement au cours d'un second épisode froid, moins accusé que le premier, suivi par une reprise de la sédimentalion.

\section{Sédimentalion de la conche zonée $C$.}

La couche (i des fig. 1 et 2 est formée de sables gris-jáune, zébrés de lits horizontaux bruns. l'épaisseur des lits bruns est très faible (2 a $3 \mathrm{~mm}$ ) tandis que les couches de sable clair intercalaire sont épaisses en moyenne de $5 \mathrm{~cm}$. Les lignes brunes sont légèrement ondulèes, frisées, et réunies, par endroits, par des anastomoses obliques. L'ensemble présente des analogies, mais également des diflérences, avec les "pseudo-rarves" que nous avons décrites au Cirque de la Patrie (H. Arimsr, in Cheynier et coll., 1963). En particulier. l'épaisseur des lits bruns est ici beaucoup plus faible.

a) Origine du sable. Le sable de la couche C s'est mis en place au cours du Quaternaire. Il est. manifestement emprunté aux sables auversiens du voisinage, ainsi que cela ressort de la granulométrie comparée du sable de la couche C de la Chambre aux Fées et des sables auversiens de la région de Chàteau-Thierry-Fère-en-Tardenois, qui ont été étudiés par M. Mrrcıfr (1948).

Ia fig. 3 montre que le sable de la couche $\mathrm{G}$ (tris homogène du haut en bas comme nous le préciserons plus loin) appartient bien à la famille auversienne de la réçion. Il est moins bien classé que la plupart de ces sables auversiens, ce qui permet d'affirmer qu'il n'est pas intervenu de phénomènes éoliens importants dans sa mise en place. Sans doute est-il descendu par un transport éolien très courl ou plutòt par une sorte d'écoulement sec, le long du flanc du coleau².

b) Zonalion. La zonation en lits alternés bruns et ocre, et la concentration préférentielle des oxydes de fer qu'elle implique, ne sont pas en rapport avec une diflérenciation dans la granulométric des sables. I.a fig. 4, où sont reproduites les courbes cumulatives correspondant à huit couches alternativement claires el ocre de la couche $\mathrm{C}$ (fig. 1), montre la remarquable homogénéité de cet ensemble. La fig. is souligne la faible variation des grandeurs caractéristiques : quartiles $\left(q^{1}\right.$ et $q^{3}$ et grossièreté ( $q^{2}$ ou g). Il apparaît donc qu'il n'y a aucune modification significative quand on passe d'une couche claire à une couche brune ${ }^{3}$.

(2) On sait qu'en climat tempéré les ruissellements sont quasi inexistants sur les sables perméables (A. Call.1:cx, $1939)$.

(3) Ces granulometries ont ite efiectures par $\mathbf{3}$ me Dubois-Page, au laboraloire de Géologie du Quaternaire du cirst, à Bellevue. 
Par ailleurs l'oxyde de fer. observé dans les sables de la couche 1, est, sous la forme d'aggolomérat: le petils cristaux, soit libres entre les grains de quart\%, soit collés par petiles plages sur la surfare des quartz. On ne décile au microscope aucune différence dans l'élat de l'oxyde de fer des couches brunes et des couches claires, en dehors d'une différence quantilative. Je n'ai pas observé d'humus dans les sables de la couche zonée. Ajoutons que l'ensemble des coupes pratiquées pour les fouilles a révélé l'indépendance de la zonation de la couche G et du pozdol. La zonation existe en maint endroit où il semble bien que le podzol ne se soil pas formét.

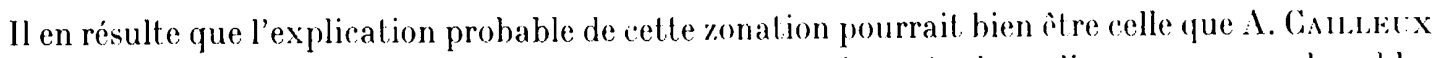
$(19(3 ; 3)$ a récemment proposée pour une zonation analogue observée dans diverses roupes de sables stampiens ou auversiens, bien que les épaisseurs des couches claires et brunes décrites par A. Cant.lirx

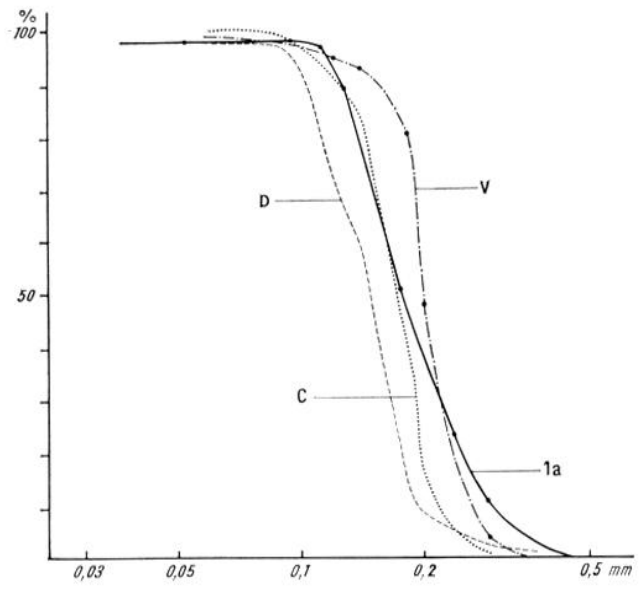

3. Cimmulomitrie comparie du sable la couche II de la chambre des fiees el des sables amversiens du Tardenois. $V$ : Vincelles, $\mathrm{l}$ : Combernon, I) : 1)huisy. Courbes cumulatives en coordomnees semilogarilhmiques. Sables du Tardemois dapres

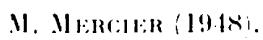

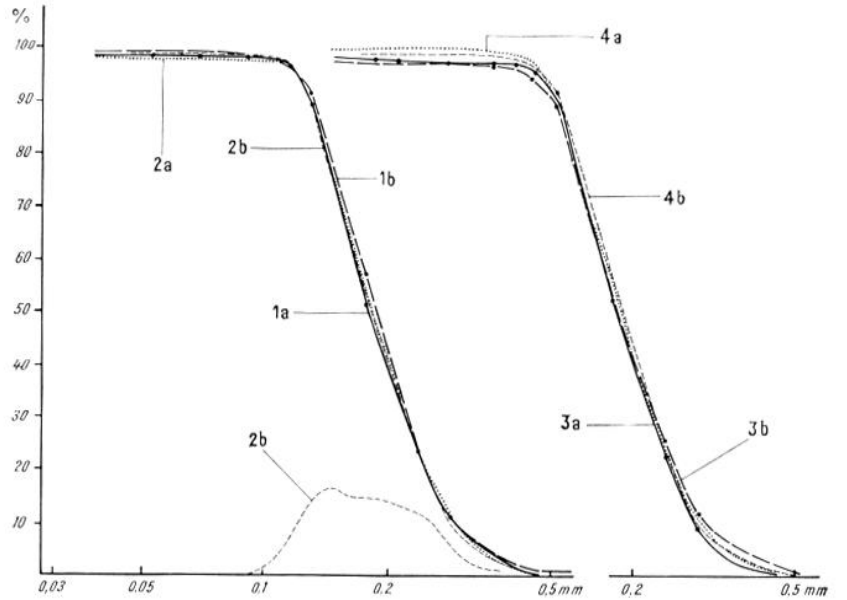

1. Gramulumelrie compande des sables des zones allermalivemenl claires el hrunes de la conche II, en coordomnes semi-logarithmiques. Coumbes emmulatives. On a trace la comble de fréguence pour le sable de la conche ?h. Pour l'emplacement des prélevements. voir la fig. 1.

soient en général netlement plus grandes. Notre confrire fait appel à des phénomènes de congélation différentielle dans les sables. la congélation de la glace en lentilles horizontales superposées précédant. la précipitation de l'hydroxyde de fer, qui se fait alors dans les espaces restés libres. I)es faits de cet, ordre ont été observés de nos jours dans le domaine boréal, notamment en sibérie el en Mlaska. Ils impliqueraient done, au moment oì ils se sont produits. a Coincy, un climat froid, peut-iblre moins rigoureux que le climat boreal artuel, si on tient compte de la faible ampletir du phénomíne a Coincy.

Il apparaît que les processus climatiques qui ont donné naissance à cette zonalion sont légirement postirieurs à l'installation lardenoisienne. En cflet, a la Chambre des Fées, en amont des coupes des tig. I ol 2. le Tardenoisien se trouve au sein de la couche zonée, Irois doublels élant observables all-dessus de l'industrie (J. Hinol:T, fig. fi et 8). Landis que dans les coupes I el. Il, il surmonte l'ensemble zoné. si l'explication que nous adoptons est exacte, apris le Tardenoisien de Coincy, le climat aurait comporté un épisode relativement seviere.

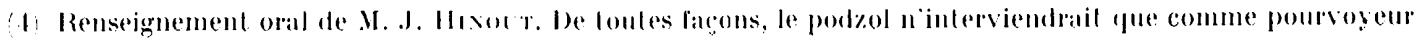
doxide de ler. 


\section{Fragments rocheurr de la couche archeolugique.}

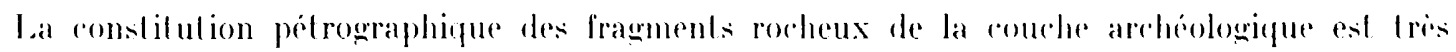
netlement différente de celle de la rouche (. Il s'agit presque exclusivement de fragments de calcaire silicific du Bartonien, auxquels ne se mèlent que trìs peu de fragments de gris auversiens. Les frayment: (jusqu'i 10 et $12 \mathrm{~cm}$ ) sont isodiamétraux en général. En outre. lous res fragments ont subi l'action du feu : plusieurs sont éclatés et les oxydes de fer y sont réduits. Le calcaire silicieux bartonien affleure au sommet du coteau. C'est lui qui a fourni la matière premiere nécessaire ì l'industrie. Les fragments rocheux de la couche archéologique ont été apportés par l'homme. Ils n'ont pas de signification géologique. Par ailleurs. on n'observe pas, à leur surface. les traces de cryoclastisme si caractéristiques des plaquettes de la couche C. Il n'y a donc pas d'indices de froid lors de l'occupation lardenoisienne.

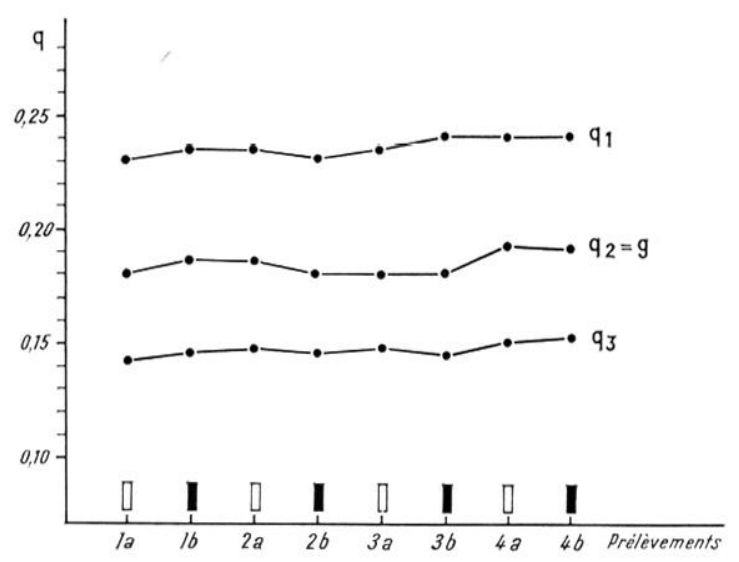

5. Variation des quartiles $q_{1}$ el $q_{3}$ et de la grossiereto qa - $x$ a travers la succession des zones claires el brun fonce de la couche 11 , de 1 a 4 . l,es petils rectangles indiquent s’il s'agit d'une couche claire on foncere. Pour l'emplacement des preflevements, voir la dig̣. I.

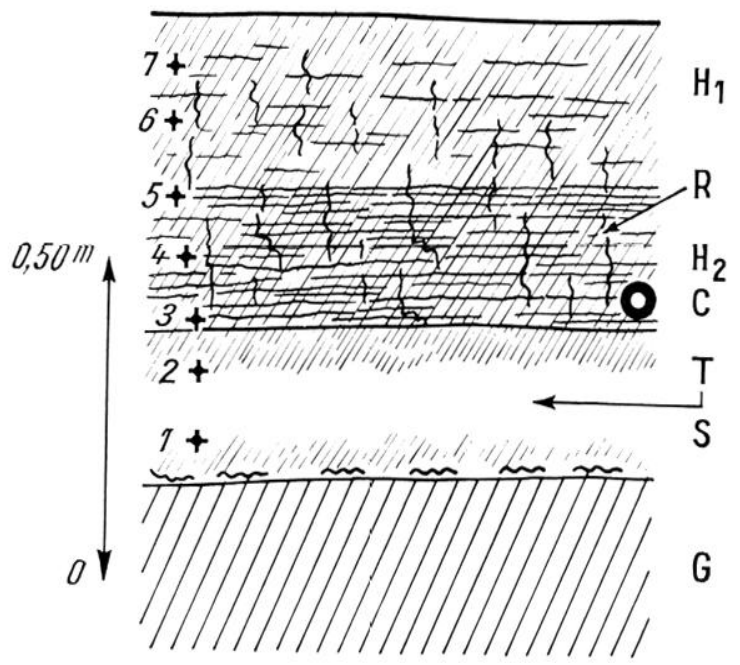

6. Coupe an Marecage-sous-le-Cieant. II : Horizon Lourbeux, noir-brun. $\Pi_{2}$ : Horizon noir tres tourbeux. $S$ : sable gris assez clair, marber de gris-brum, surtoul en haut de la couche. ( $i$ : bloc de gres enfoui. $R$ : racines. \% : Place de l'indust rie lardenoisieme. $1:$ : prelivement pour la radiochronologie. A gauche, les croix indiguent la place des prelievements pour l’elude palynologique.

\section{Le portzol}

I'n podzol dont les horizons $\Lambda_{1} . \Lambda_{2}$ et 13 sont assez bien individualisés. occupe les $0,80 \mathrm{~m}$ supérieurs de la coupe I (fig. 1). L'horizon $\Lambda_{0}$, non figuré, n'étail pas présent, enlevé sans doule par des fouilles antérieures. $A_{1}$ est grisâtre. $\lambda_{2}$ est typiquement blanc. B est noir, marbré de jaune. Il contient le niveau archéologique vers sa partie haute.

Sur la coupe 11 , le podzol est un peu moins épais $(0.60 \mathrm{~m})$, les horizons $\mathrm{A}$ sont réduits, $B_{1}$ et $B_{2}$ sont bien distincts, $\mathrm{B}_{2}$ étant ici un alios véritable. brun, foncé, dur (fig. 2). Lin ce point c'est lui qui conlient la couche archéologique.

La formation de ce podzol est postérieure à la zonation ${ }^{5}$. postérieure à l'occupation tardenoisienne, et à l'enfouissement des silex tardenoisiens sous une couche de sable alteignant $0.50 \mathrm{~m}$ d'épaisseur. La station de Coincy apporte done, quant à la chronologie des podzols, une donnée intéressiante. les processus pédologidues y élant netlement postérieurs a l'orcupation lardenoisienne.

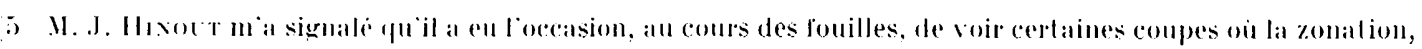
implique dans la couche perdologique. se distingue encore, bien que fablement, dans laalios. 


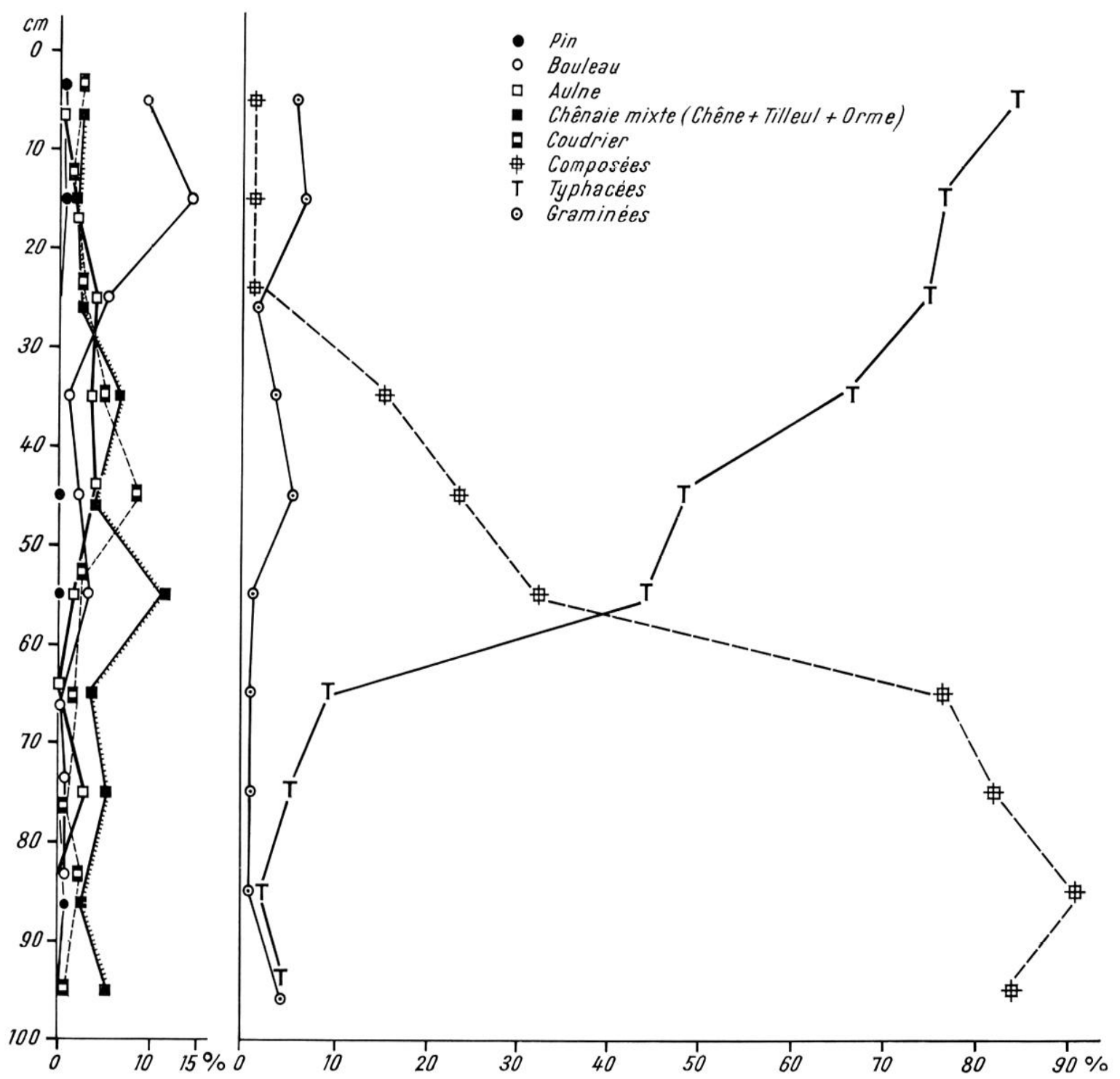

7. Diagramme pollinique de la chambre des Fées coupe Ij. A ganche, arbres el arbustes, à droite, flerbacies. Seules les formes essentielles sont représenties.

L'examen des fails géologiques de la slation tardenoisienne de Coincy permet de mettre en évidence, au point de rue de la chronologie géologique, la succession suivante.

phase de sédimentation sableuse;

période froide, de fort cryoclastisme (iclatement superficiel des gris a peau de rrocodile); arrilt de la sédimentation. Période de faible éolisalion el d'acequisition d'une mince patine grise ;

période de léger cryoclastisme ;

-.. phase de sédimentation sableuse, au cours de laquelle se produit l'occupation lardenoisienne (sans indice de froid), puis formation de la zonation, vraisemblablement liée à un épisode froid ;

-- podzolisation.

L'étude sédimentologique ne permel pas d'aller plus arant dans l'analyse climatique et chronologique de la station tardenoisienne de Coincy-en-T'ardenois. 


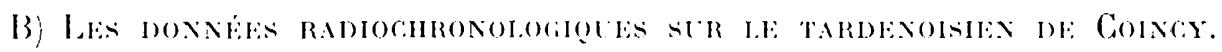

Au cours de ses fouilles a Coincy, J. Hixol a prélevé trois échantillons susceptibles de fournir une datation par la méthode du radio-carbone.

1o des fragments de charbon de bois. inclus dans la couche tardenoisienne de la Chambre des Fées ( 19 du plan horizontal, J. Hixour, 1964, a (6i) cm de profondeur).

$2^{0}$ des fragments de charbon de bois, provenant du foyer $n^{\circ} 2$ de la Chambre-des-Fées (J. IInovt, 1964) a 15 $\mathrm{m}$ de distance du site précédent, inclus à une profondeur de $70 \mathrm{~cm}$, dans la couche à industrie tardenoisienne.

$3^{0}$ un échantillon de tourbe, au lieu-dit le Marécage-du-(iéant (roupe fig̣. 6), prélevé vers la base du niveau tourbeux superposé à l'industrie tardenoisienne.

Les déterminations ont été eflectuées au Iaboratoire du Radiocarbone de (ifi-sur-Yvelle, el

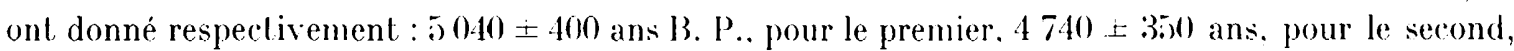
el $3260 \pm 200$ ans pour le 3e échantillon. La grande imprécision sur l’àge indiqué pour les échantillons en provenance de la Chambre des Fées tient a la faible quantité de charbon disponible. dans les deux cas, pour les mesures d'activilé. I a méthode de date décrite par ailleurs (G. I)nummas. M. T. Proncis, 1958), utilise un compteur proportionnel de 1 litre de volume, rentipli a la pression atmosphérique de gaz rarbonique.

Les deux premières déterminations datent le Tardenoisien de Coincy de la partie terminale des Lemps atlantiques, Landis que la base de la lourbe du Marécage-des-(iéants, postérieure à l'indusIrie, appartiendrait a la fin du subboréal. De tels résultats ont paru au premier abord tout a fait, surprenants. Si on ne posside, jusqu'ici. a notre connaissance, aucune date pour le Tardenoisien français. plusieurs datations ont été données pour le Tardenoisien de Iollande et de Belgique (A. Bommes et A. (). Woxtris, 1957). Elles sont toutes comprises entre 6960 et 7970 B. P., ce qui rapporte ces industries aux débuts des temps atlantiques. Cn résultat de mème ordre parail ressortir d'une étude faite sur un sile de sloraquie, dont la flore appartient au début des temps atlantiques. el l'industrie a la limite Tardenoisien inférieur-supérieur (J. BAn'A, 1957). Par ailleurs, on sait que l'Ahrensbourgien débute au Préboréal (9500 et, 9:310 上260 à stellmoor. Ilolstein), mais il subsiste jus(qu'à a $340=200$ dans la mème station. L'industrie d'Ertebolle-Fillerbek, à satrup Moor, Rüde. Schleswig-Holstein, altribuable al Mésolithique final ou au Prolonéolithique, a donné lieu i de nombreuses datations, qui ront de 6060 a 5620 : $200^{6}$. Enfin, rappelons que le Néolithique de Biarritz, sous son facies asturien, remonte à 5340 上 200 (F. Ondmisto, 1960).

Ainsi le Tardenoisien de Coincy serait plus récent que toutes les industries mésolithiques de l'Europe septentrionale et contemporain des premieres industries néclithiques. Devant de telles conclusions, devons-nous douter du résultal de la radio-chronologie? Il nous reste à interrocrer le contenu sporopollinique des couches de Coincy et à demander a l'analyse pollinique des arguments susceptibles de lever cette incerlitude.

\section{G - PAisologil.}

\section{Introduction.}

L'analyse sporopollinique a été effectuée pour 4 séries d'échantillons, prélevés respectivement sur une mème ligne verticale. d'une part le long des deux coupes 1 et 11 du lieu-dit Chambre des F'ées d'autre part au Marécage-sous-le-Géant et enfin, au sommet du versant, à la partie haute de l'aflleurement auversien, au lieu-dit la Hottée-du-Tiable. Les points précis des prélevements de chaque coupe, généralement dislants de $10 \mathrm{~cm}$, sont indiqués sur les figures (fig. I, 2 el 6 ).

6 Ces diverses dates onl de releves dans le fichier public par " hadiocarbon dales Association, incorporated, Andover, Jassachussets $n$. Toutes les dales cites dans celte note sonl compties avant lepoque actuelle. 


\section{Irépiaralion des ichantillons.}

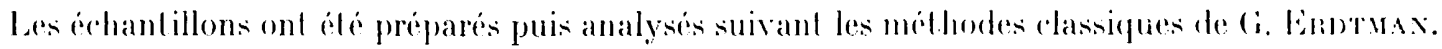
Truilement chimique : Fll $70 \%$ à froid. Loll $10 \%$ a chaud, chloral ion, acélolyse.

\section{Liésullats el interprétalions des analyses sporopolliniques.}

Les échantillons se sont avérés très riches en grains de pollen. La fréquence relative ou nombre de pollens et spores dénombrés sur une mème surface de préparalion égale i $32 \times 22 \mathrm{~mm}$ a ćté souvent de plusieurs centaines dépassant parfois le millier, au Marécage-sous-le-(réant. Les résultats les plus intéressants nous ont élé domnés par la coupe I de la Chambre des liées (fig. 1) el par relle du Marécage-sous-le-(jéant, (fig. 6).

Les différents pourcentages des Familles. (ienres ou bispires reronnus sont groupes en lableaux. Chaque pourcentage a été évalué par rapport i l'ensemble des formes sporopolliniques. I es variations de la silve et des herbarées sont fiqurées par des diagrammes.

1) "Chambre des Fóes" . coupe I (fig. 7, labl. J!. Les cinq niveaux les plus inférieurs (1 m a $1,50 \mathrm{~m}$ ) ont livré trop peu de grains de pollen pour permeltre l'établissement de pourcentages valables. Ces résultats sont consignés dans le tahleau I mais la représentation graphique n'a pu ilre commencée qu'au niveau $6(0,90 \mathrm{~m}$ a $1 \mathrm{~m})$.

La silve (arbres el arbustes) est constamment subordonnée aux herbacées. Parmi les arbres, la Chènaie mixle. aver Tilleul dominant tient lat premiere place. Lille s'efface au niveau $11(0.40 \mathrm{~m}$ a $0.50 \mathrm{~m}$ ) devant le Coudrier. et a parlir du niveau 1.3 (0.20 m a $0.30 \mathrm{~m}$ ) devant le Bouleau qui devient préponderant en surface. I'n seul pollen d'Orme pst présent au niveau 1.1. Le Pin. Le Fresne el le Charme sont sporadiquess le Hitre tres rare.

Les herbacés dominent lout au long du diagramme. Eilles sont essentiellement representiess par des Composées el des Typhaceses secondairement par des (iraminées. Nous notons ígalement. la presence de pollens d'/ler el autres . Iquifoliacées, ainsi que relle plus rare de Planlaginacées, Chénopodiacreses (. Hriplerr). Caryophyllarées, Oléarées. Polygonarées. liosacées, Ombellifires. Légumineuses et Araliacées (Iledera helir). Les Composees sont tres importantes jusqu'au niveau 10 (0.5) i 0.60 mi a part ir dupuel elles régressent brusquement au profit des Typhacées. Ce niveau 10 marque le début d'une augmentalion de l'humidite qui ne cessera de croil re. comme en lémoignent les pourcentages de plus en plus álevés de pollen de Typha. Pendanl la croissance de sa courbe, la silve est marquée par une légire prépondérance du Coudrier, puis par celle du Bouleau.

Les spores sont peu importantes. Nous ne notons la présence que de rares Fougères ( $\boldsymbol{P}$ oblypodium vulgare et .lthyrium).

2) "Chambre des liés"s. coupe II. Kous n'avons pas figuré le lableau des pourcentages et le diagramme correspondant a la coupe II de la chambre des Fés. celle-ci élant perlurbépe par la présence d'un gros bloc de grese enfoui. Neanmoins, dans leurs grandes lignes, les varialions polliniques sont identiques it celles de la coupe l. La silve est semblable aver poussée du Coudrier a la hase. sous le rocher, et. le Bouleau prépondérant en surface. Les Composées et les Typharcés dominent, l'ensemble, arcompagnées par les Ciraminées.

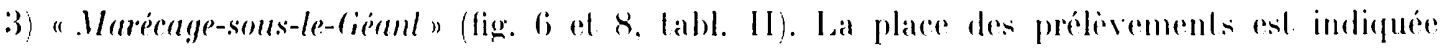
sur la fig. 6. qui donne la coupe au "Narécage-sous-le-créant".

Silne. I l'exception du Coudrier qui est prépendérant dans la partie médiane du diagramme.

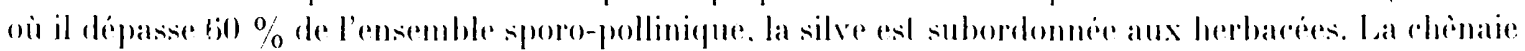
mixle, formée essent iellement par le Chene areompagne du Tilleul, est ronstante ainsi que le Bouleaul. Le Pin est loujours présent mais peu abondant. L.Sulne croit legerrement en surface. Le firesne, les Charme et le Hetre sont rares et sporadiques. 

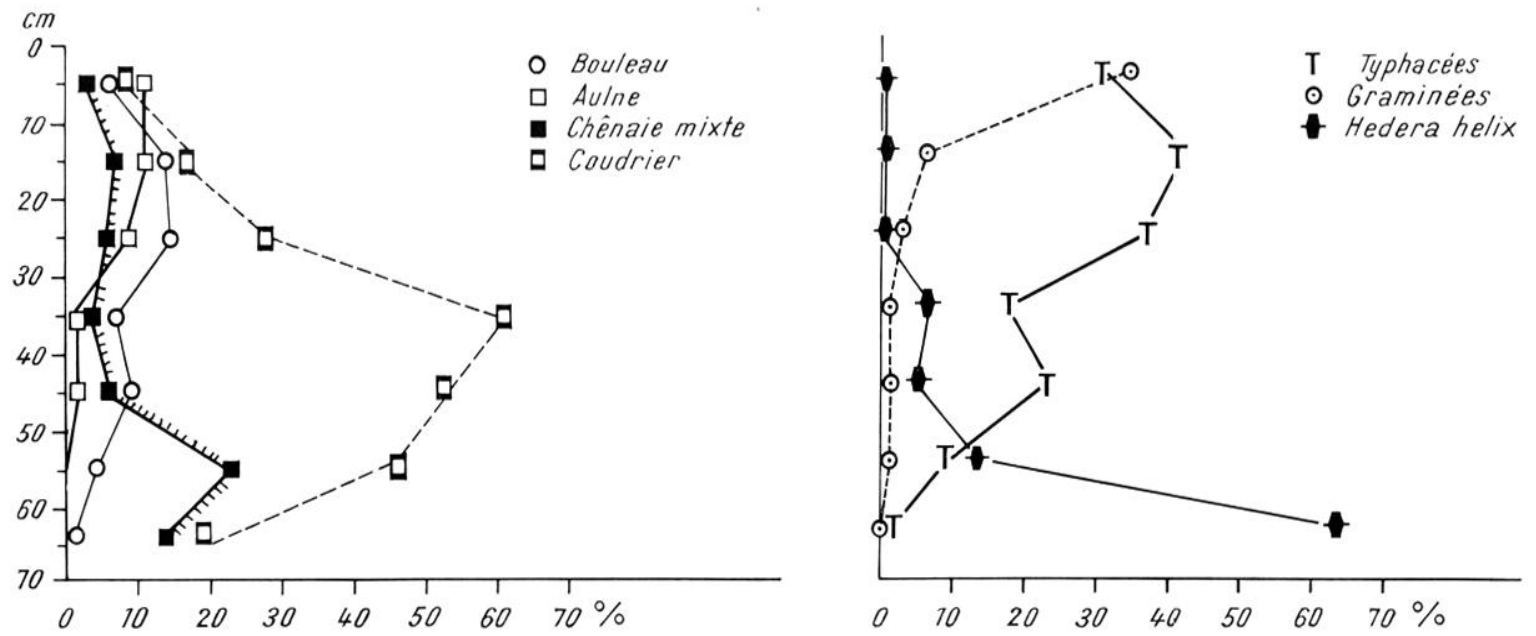

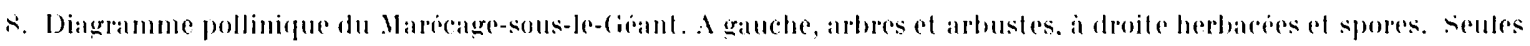
les formes pessentielles sont representies.

Ilerbacées. Dans le niveau de base. le pollen d'Iedera helix est nettement, dominanl (6:3 \%': il régresse ensuite au profit du Coudrier et des Typhacées. Les Graminées dépassent 30 \% en surface. Les Composées sont rares ici. ainsi que d'autres pollens d'Herbacées telles : les Chénopodiacéen (.Itriplex), Caryophyllacées. Aquifoliacées, Oléacées. Ombellifères, Légumineuses, Plumbaginacées, (.1rmeria vulgaris) et Dipsacées (Scabiosa).

Les spores de Ptéridophytes sonl peu importantes; parmi elles nous avons pu déterminer des Polypodium mulgare, Athurium. Driopteris, Osmunda et Selaginella.

1: La " Iollée du Diable ". Quelques prélèvements ont été effeclués aux fins d'analyse pollinique, au sommet des sables de l'Auversien, au lieu-dit "La-Hottée-du-1)iable». Seuls les niveaux supérieurs remaniés ont livré des pollens. La silve quaternaire. trouvée ici, est formée essentiellement par des Tilleuls, puis des Chènes el quelques Bouleaux, Aulnes, Pins et Coudriers. Elle est subordonnée aux ILerbacées (Typhacées et (iraminées). Les niveaux tertiaires plus profonds. non remaniés, ne renfermaient aucun pollen. L'absence de pollens lertiaires permet d'envisager romme improbable leur présence. mèlée aux pollens qualernaires, dans les sédiments de la "Chambre des Fées" el du "Marécage-sous-le-Giéant ".

\section{Conclusions.}

lin considérant les varialions silvaliques, mises en évidence par le diagramme de la Chambre des Fées (coupe I) nous observons. il la base, une Chènaie mixte avec Tilleul bien représenté. parfois mème dominant, accompanné de Coudrier. Cette phase, avec peu ou pas de Pin, évoque pour nous la fin de la période allantique (J. Sacva(is, 1954). Elle est suivie, a partir du nivean 13, par une période plus froide à Bouleau dominant qui correspond au subboréal, phase souvent marruée

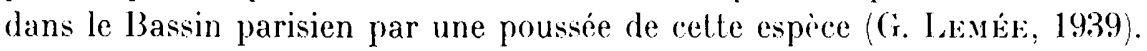

Par la radiochronologie la date du niveau 10, renfermant. l'industrie tardenoisienne, est déterminée. Yous savons que ce niveau correspond a i) 040 B. P.. ce qui est parfaitement en accord avec notre datation "fin Atlantique " auquel fait suite le subboréal et peut-ètre aussi le subatlantique. la silve de ces deux dernières phases étant souvent peu différenciée dans le Bassin parisien.

l)ans le diagramme du Marécage-sous-le-(iéant, nous datons également par le Carbone 1.4 de 3960 B. P. une couche située, entre les prélerements 3 et t. Cette date correspond approxima- 
livement à la limite Subatlantique-siubboréal. La silve avec Bouleau est bien celle qui caractérise cetle période. Ici, l'indlistrie tardenoisienne se silue entre les niveaux inférieurs 1 et 2 , lesquels correspondraient palynologiquement, comme à la Chambre des Fées, à la phase fin dllantique précédant, la Subboréal.

Notons que dans chacun des deux diagrammes figurés, le Tardenoisien est accompagné d'une poussée du Coudrier. Fille est moins importante à la Chambre des fiées, mais cependant nettement, marquée. Nous avons également une humidité certaine des lieux, prouvée par la courbe croissante des Typhacées; elle peut itre un reflet climatique strictement local; nous reviendrons sur cette question par la suite.

I còté de ses aspects très significatifs de l'ensemble de la silve de Coiney, quelques considérations, de moindre importance, se déduisent de l'examen des herbacées et de quelques arbres. Ainsi ia la Chambre des Fées, les Composées puis les Typhacées sont très nombreuses tandis qu'au Marécage-sous-le-Géant le Coudrier est nettement prépondérant --- exception faite pour le niveau de surface, les Composées sont rares et nous avons rencontré, à tous les niveaux, des pollens d'Iledera helix ceux-ci sont très nombreux dans le niveau de base. Étant donnée la fréquence très élevée des grains de pollens contenus dans nos sédiments, nous pensons à juste titre qu'ils sont, en grande partie, le reflet d'une végétation fossile " in situ ». I'ne humidité locale des lieux a permis l'installation de lierre à l'endroit précis du Marécage-sous-le-(réant, comme elle a permis l'installation de Typhacées el leur grand développement à la Chambre des l'ées. Quant aux différences dans les pourcentages de Coudrier, elles peuvent s'expliquer par le fait que cet arbre ì chatons dissémine localement ses pollens en plus ou moins grande abondance.

Toutefois ces aspects locaux ne masquent pas les traits essentiels de la silve de Coincy qui permeltent de placer sans ambiguilé l'évolution végétale de celte région dans les cadres classiques de l'liurope moyenne.

Les datations obtenues par la radiochronologie sont en accord avec les résultats palynologiques, mais elles le sont moins avec l'âge arcordé jusqu'ici à l'industrie tardenoisienne, mise couramment en parallèle avec le Préboréal el le Boréal, c'est-i-dire avec une phase de végétation qui devrait ètre caractérisée par l'abondance du Pin (J. satvacib, 1954). Evidemment, le fait d'envisager que l'ensemble du Marécage, avec ses pollens tris nombreux, reflite surtout la végétation fossile "in silu " pourrait inciter ì penser que le Pin (pré-boréal ou boréal) n'a pu se développer sur des lieux trop humides; mais si ce pollen à saes aérifères, facilement transportable, existait aux alentours, nous en aurions davantage de vestiges dans nos sédiments. Or nous avons constaté (J. SAuvacir, 19\%4, p. 26) que, dans la vallée de l'Ourcq, à moins de $10 \mathrm{~km}$ de Coincy, le Pin est présent et très abondant (plus de $70 \%$ ) au cours de la phase boréale.

Il nous semble plus plausible de rester en accord avec les datations données par les dosages du Carbone 14. Il est d'ailleurs éclairant de faire un rapprochement avec la palynologie du site archéo-

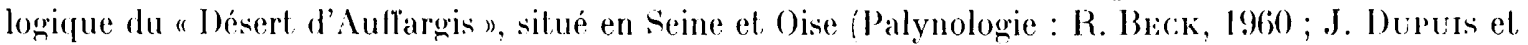
R. Brok 1961). La, un niveau lardenoisien avail également, livré des pollens de l'extrème fin de la Chènaie mixte. avec Tilleul abondant; secondairement, venaient le Coudrier, l':Lulne. le Bouleau et le Hetre. Getle phase était suivie par une poussée du Coudrier ì laquelle succédait une recrudescence du Bouleau acrompagnée d'une timide apparilion du Pin. Malgré la quasi absence de ce pollen, J. I) Pros avait maintenu le 'Tardenoisien dans le Préboréal-Boréal et expliquail la présence d'une flore "fin Atlantique" a ce niveau par une descente des pollens. Avec le seul exemple d'Auftargis, cette hypothise pouvait ètre retenue plus facilement que maintenant où nous serions obligés d'avancer aussi des conclusions identiques pour la Chambre des liées et le Marécage-sous-le-Géant. Cette descente de grains de pollen au travers les sables nous parait actuellement bien peu probable, car il faudrait alors supposer qu'elle ail été faite partout d'une façon systématiquement, régulière, les diagrammes d'Auffargis et de Coincy restant dassiques; or ceci est assey diflicile a envisager. Il 


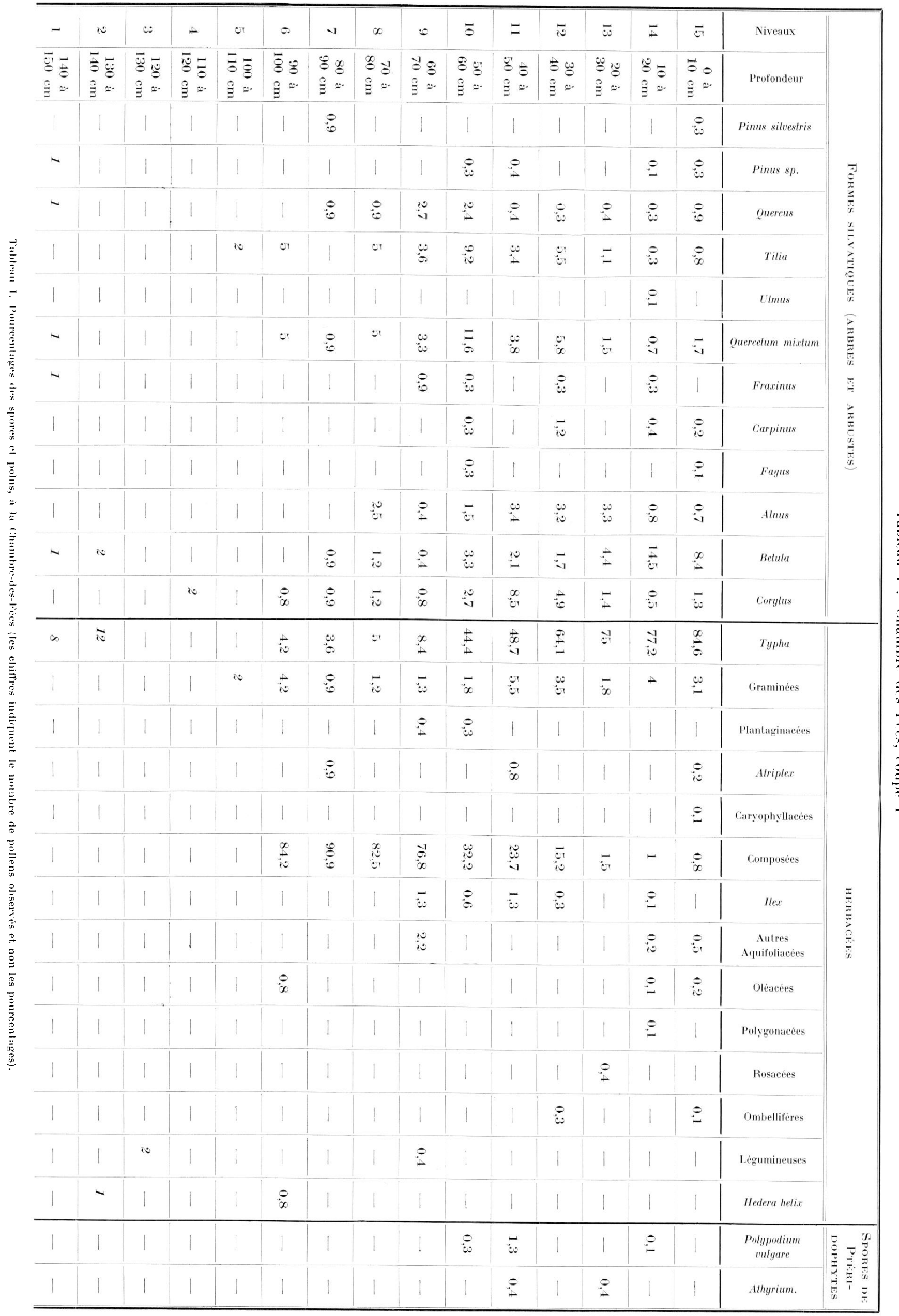




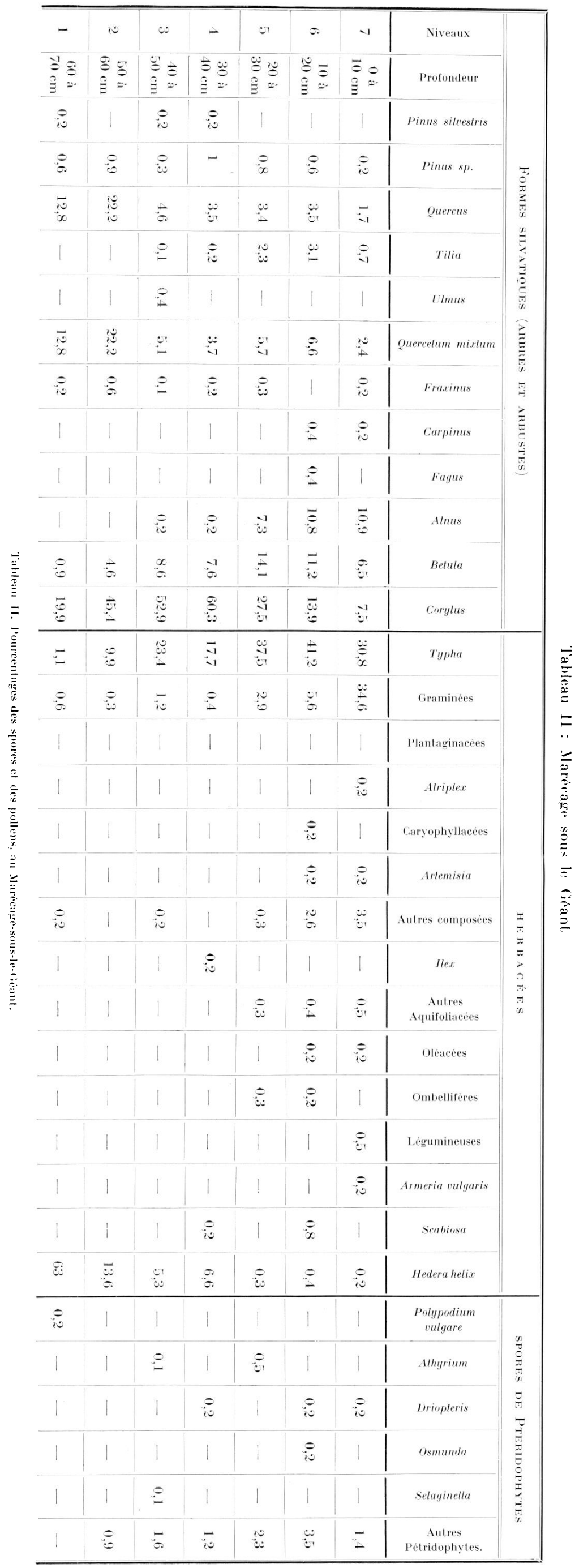


semble beaucoup plus nomal de meltre en parallele les qisements de la Chambre des fées. du Marécage-sous-le-Géant et celui du Désert d'Sullarqus pour conclure à l'existence d'un Tardenoisien lardif a la fin de l'Mllantique.

\section{1) CoNetrans.}

L'étude géologique, radiochronologique el palynologique de la slation Lardenoisienne de Coincy. apporte une série d'arguments concordants, qui conduisent aux conclusions suivantes.

L'occupation tardenoisienne a coincidé avec une période de climat tempéré et humide. Eille a été précédée de plusieurs épisodes de cryoclastisme, l'un trés fort, l'autre plus modéré. séparés par une phase d'éolisation. L'époque tardenoisienne a été suivie d'un seul épisode froid, de lype modéré. Ces données géologiques el sédimentologiques sont prérisées par l'analyse palynologrique. Celle-ci, qui a porté sur les couches postérieures aux épisodes de cryoclastisme et d'éolisalion antétardenoisiens, rapporte la flore tardenoisienne i la fin de l'Atlantique. Ainsi. dans les épisodes froids anté-Lardenoisiens, on pourrait voir la trace des dernières époques à Ir yas et du Boréal. Landis que l'épisode de froid modéré, post-tardenoisien. serait sub-boréal. La radiochronologie, dalant les charbons tardenoisiens de $5040 \pm 400$ et 4740 上3:0 B. P., apporte une indication cohérente aver cess déductions.

Ces données paraissent aberrantes, au milieu des faits acquis pour le Mésolithique de l'Lurope septentrionale. Cependant. la station tardenoisienne du Désert d'Auffargis, dans la région parisienne. a elle aussi une flore de la fin de l'Atlantique. On ne peut que ditlicilement admettre une telle concordance, s'il s'agit dans les deux cas, de faits aberrants. Il nous parait sage, cependant, de laisser encore la question ouverte, jusqu'a re que de nowelles études palynologiques el radiochronologiques viennent infirmer ou confirmer nos résultats.

Vous soulignerons également l'intérèl de la station de Cioincy quant à la datation de la podzolisation. Ce phénomène, évidemment postérieur a la mise en place des sables les plus élevés du gisement, est donc ici relativement récent, postérieur a la date extrème fournie par le diagramme pollinique de la coupe I, c'est-a-dire subboréal ou postérieur.

\section{H. Mlintex. (i. Deimbias et J. Salvagr:}

BHRIOKRAPHIE

Almux (H.) Considérations géologiques sur la station prohistorique du Cirque de la latrie, près de Nemours, in

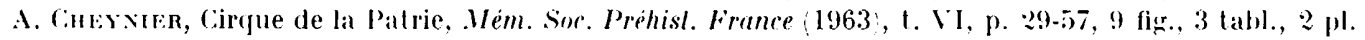

Barta (J.), les dunes pléistocines des environs de sered, leurs industries palélithiques ef mesolithiques, Slocenstia Archeologia, t. 5, Bratislava, 1957,21 fig., $22.2 \mathrm{pl}$.

Black (R.), Étude palynologique de deux gisements de la région parisienne : le "Cirque de la Patrie "... le "Diserl d'Auffargis ». Dipl. Et. Sup., Strasbourg, 1960, 27 p. dactyl., 1 fig., 6 diagr., 4 tabl., 3 cartes.

Bonmers A.) el Woxtiks (A. O.), Statistics and graphs in the study of Flint Assemblages, Palaenhishria, rol. V. $(1957)$, p. $27-28$.

Brocur (M.), Morphologie de deux régions arëigues du Bassin de Paris el leur relation arec le Périglaciaire, thèse, Fac. Sc. Paris, 1955, ronétype, 15.4 p., 19 fig., XI tabl., VII pl.

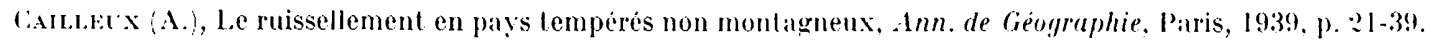

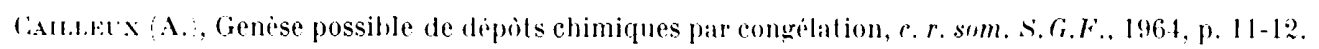

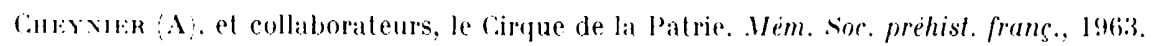




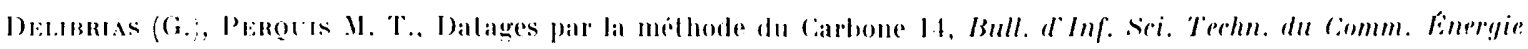
Alom., $n^{\circ}: ? 1,193 \pi, 8.1-3$.

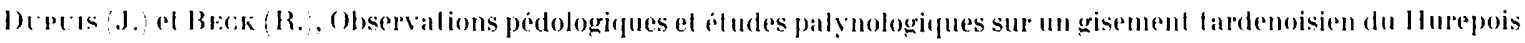

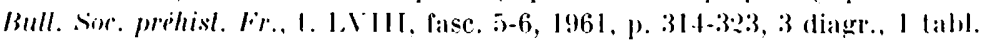

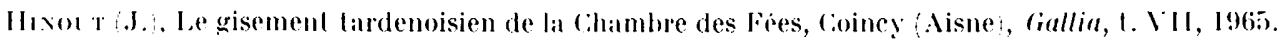

Lavie: (a.) Recherches sur l'histoire forestiere postglaciaire de la Basse-Normandie ef du Perche, Bull. sime. Limn. Vormandie, (aen, 9e série, 1. 1, 1939, p. 97-145.

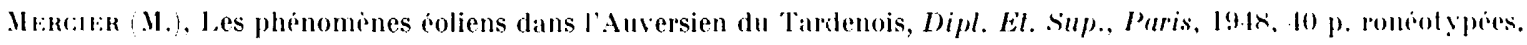

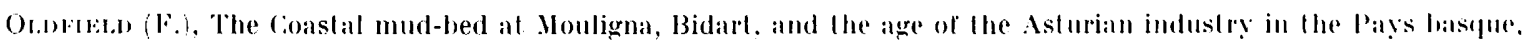
Pollen el spores, $1 .: 2,1960), 11^{\circ} 1$, p. 57-70, 4 fig.

SAuranie (J.), Palynologie et petrographie de tourbes el de sédiments de la lavelle parisienne of des Ardennes, Thise,

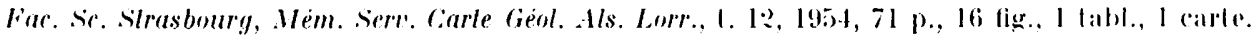

